\title{
Review \\ Pro/Con debate: Should 24/7 in-house intensivist coverage be implemented?
}

\author{
Yaseen Arabi
}

Intensive Care Department, King Saud Bin Abdulaziz University for Health Sciences, King Abdulaziz Medical City, ICU 1425, PO Box 22490, Riyadh, 11426 K.S.A.

Corresponding author: Yaseen Arabi, arabi@ngha.med.sa

Published: 5 June 2008

This article is online at http://ccforum.com/content/12/3/216

Critical Care 2008, 12:216 (doi:10.1186/cc6905)

(c) 2008 BioMed Central Ltd

\begin{abstract}
You are appointed director of a new large multi-discipline intensive care unit in an academic center. The hospital is affiliated with a medical school and as such there will be an adequate number of medical students, residents, and fellows (specializing in critical care) rotating through the unit. The unit will be a 'closed' (intensivist-led) model. In setting up the call schedule for the intensivists, you need to decide whether the mandate will be for the intensivists to provide $24 / 7$ in-house coverage as opposed to off-hour coverage from home. You wonder about the sustainability of each model.
\end{abstract}

\section{Pro: 24/7 in-house intensivist coverage should be implemented}

Intensivist coverage traditionally has followed the 'business hours' model used in other professions, with reduced availability during weekends, weeknights, and holidays. This type of staffing would be appropriate if the need for the service were limited to business hours or if delaying the service (for example, from the night to the next morning) did not have any negative consequences. Clearly, this is not the case in critical care. First, critical illness does not recognize the boundaries of business hours, and therefore qualified intensivists need to be available around the clock. Studies have demonstrated that $66 \%$ to $69 \%$ of intensive care unit (ICU) admissions are admitted during off-hours [1,2]. Second, in no area more than in the ICU is the outcome of patients affected by providing the right treatment at the right time; delays in such treatment have been demonstrated to have negative consequences [3-5].

Several studies have demonstrated increased mortality of acutely ill patients admitted during weekends, weeknights, and holidays, a phenomenon that has been attributed, at least in part, to lower staffing levels. A large Canadian study showed a significantly increased risk of death for patients admitted during weekends with several acute diagnoses, including ruptured abdominal aortic aneurysm, acute epiglotitis, and pulmonary embolism [6]. A study from California showed higher adjusted mortality for patients admitted from the emergency department on weekends compared with those admitted on weekdays [7]. Of note, a larger 'weekend effect' was observed in major teaching hospitals [7]. Similarly, a Finnish study showed that weekend and weeknight ICU admissions were associated with increased mortality even after adjustment for severity of illness [8]. In a multi-center pediatric ICU study, emergency admissions during evening hours had a higher mortality, especially for patients admitted with shock, congenital heart disease, or after cardiac arrest [9]. Investigators from the Cleveland Health Quality Choice program found higher mortality for weekend admissions compared with midweek (Tuesday-Thursday) which could not be explained by differences in severity of illness [10]. Data from the Mayo Clinic (Rochester, MN, USA) showed higher mortality for weekend surgical admissions even after risk adjustment [11]. Lower staffing level during off-hours was suggested as an explanation for the worse outcome during off-hours. In support of this explanation, a recent study of myocardial infarction admissions in New Jersey from 1987 to 2002 demonstrated that weekend admission was associated with fewer invasive procedures and higher adjusted mortality [12]. The mortality difference became non-significant after additional adjustment for invasive cardiac procedures, suggesting that reduced access to care on weekends was responsible for the mortality difference [12].

Experience in treating complex ICU patients is an important determinant of clinical outcome and resource utilization [13] and there is evidence to suggest improved outcomes when care is delivered by intensivists compared with in-training physicians. Pollack and colleagues [14] studied the associa- 
tion of care factors and survival from the pediatric ICU in 5,415 admissions to 16 sites. The investigators found that admission to an ICU located in a teaching hospital was associated with reduced probability of survival. They found also that admission to an ICU with intensivists was associated with improved probability of survival. Post hoc analysis indicated that the higher severity-adjusted mortality in teaching hospitals may be explained by the presence of residents caring for ICU patients [14]. Training programs also seem to increase resource utilization. A study of 10,900 patients from Project IMPACT found that the presence of critical care fellows was associated with a significant increase in ICU length of stay after adjustment for confounding factors [13]. Additionally, several of the studies that showed worse outcomes on weekends and nights were conducted in academic centers with fellowship programs [11]. In fact, a larger 'weekend effect' of increased mortality was observed in major teaching hospitals [7].

Recent evidence suggests that having continuous on-site coverage by qualified intensivists helps in ensuring consistency of care. Our ICU is a tertiary-care academic ICU that is covered $24 / 7$ by in-house board-certified intensivists. The daytime intensivist makes the overall care plan and provides coverage throughout the week to maintain continuity of care. Endorsements at the beginning and the end of the night shifts ensure communication of the care plan to the on-call intensivists. Our data demonstrate that the outcomes are similar for patients admitted during weekdays, weeknights, and weekends [1]. Similar findings were found in a French study of 23 ICUs in which $24 / 7$ in-house coverage was provided by a board-certified intensivist or an experienced intensivist intraining (who had to have completed 52 on-site night shifts as a resident) [2]. Jacobs and colleagues [15] described the results of implementing 24/7 in-house intensivist coverage in a cardiothoracic unit of a tertiary-care teaching hospital and reported a major reduction in the risk-adjusted mortality for coronary artery bypass graft surgery from $6.25 \%$ to $1.6 \%$ with a significant reduction in ICU length of stay. Gajic and colleagues [16] reported a before-and-after study of the impact of developing 24/7 in-house intensivist coverage in the ICUs of the Mayo Clinic. The study showed improved processes of care, improved staff satisfaction, and decreased ICU complication rate and hospital length of stay [16]. Similarly, Hixson and colleagues [17] examined the outcomes of patients admitted to a pediatric ICU staffed $24 / 7$ by inhouse pediatric intensivists. The investigators found that, after controlling for important clinical differences, neither weekend nor evening admission had a significant independent effect on mortality risk.

Recognizing the impact of intensivists on patient care and efficiency, the American College of Critical Care Medicine and Society of Critical Care Medicine have recommended $24 / 7$ intensivist coverage as the ideal model for the provision of critical care $[18,19]$.
In summary, critically ill patients admitted during off-hours should get the benefit of being managed by an in-house intensivist, who is more likely than in-training physicians to make the right decisions in the first critical hours of ICU admission.

\section{Con: 24/7 in-house intensivist coverage should not be implemented}

Advocates of $24 / 7$ in-house intensivist coverage base their arguments on the presumption that adverse outcomes during off-hours are related to lower staffing levels. However, such adverse outcomes may not necessarily be related solely to differences in intensivist staffing but may also be related to lower levels of staffing in nursing, respiratory therapy, or other medical and non-medical ancillary services or to lower level staffing of 'upstream' departments where the ICU patients originated from. Additionally, worse outcomes of patients admitted during off-hours may be related to greater severity of illness. In a study from the UK, crude mortality was higher for weekend admissions and remained after adjustment for the UK APACHE (Acute Physiology and Chronic Health Evaluation) II. However, after adjustment to APACHE II components, the differences became insignificant [20]. A study from the Mayo Clinic found higher crude mortality for weekend admissions. However, the mortality difference became insignificant when adjusted to APACHE III for medical and multispecialty (but not surgical) ICU patients [11].

Additionally, the advocates of $24 / 7$ in-house intensivist coverage note that in-training physicians provide care that is inferior to that of intensivists; therefore, the former should not take a central role in managing critically ill patients during off-hours. However, this notion may not be entirely true; the higher mortality observed in teaching institutions may not be related to care delivery by in-training physicians but rather to greater severity of illness. Zimmerman and colleagues [21] have demonstrated that teaching hospitals care for more complex patients and achieve better riskadjusted survival rates. However, these hospitals have higher production cost, which is driven in part by prolonged length of stay.

In fact, good outcomes can be achieved by having experienced in-training physicians as demonstrated in the French study which included ICUs covered by either intensivists or in-training physicians [2]. Admittedly, because the study did not compare ICUs with consultants to those with in-training physician coverage, firm conclusions cannot be reached on this issue. Furthermore, the French system includes direct admissions to the ICU from the community by the French Emergency Medical Service, in which the switchboard is staffed by physicians and the ambulance team is led by a physician (anesthesiologist or general practitioner trained in emergency medicine). Therefore, even if some of the ICUs are staffed by in-training physicians, other experienced physicians are involved in the care from the onset. 
Does having 24/7 in-house intensivist coverage improve patient outcomes? The intuitive answer is yes. However, the supporting evidence is based mainly on a few observational studies. In fact, concerns about continuity of care with shift work have been raised [22].

Manpower requirements of 24/7 in-house coverage are significantly higher than the traditional coverage system. Depending on the coverage arrangements, it is estimated that 5 full-time equivalents (FTEs) are required to provide 24/7 inhouse coverage by one intensivist, and this number may be significantly higher in large ICUs with multiple teams. The severe international shortage in qualified intensivists makes the wide implementation of this model challenging at present and even unlikely in many non-tertiary-care hospital ICUs in the near future. A study of 5,980 ICUs in the US estimated that in-house physician (of any level) coverage was provided in only $20 \%$ of ICUs during weekend days, $12 \%$ during weeknights, and $10 \%$ during weekend nights [23]. The study also found that only $4 \%$ of all adult ICUs met the full Leapfrog standards (a high-intensity ICU staffing pattern plus dedicated attending coverage during daytime plus dedicated coverage by any physician during nighttime). A Canadian survey found that dedicated in-house physician coverage overnight was available in only $60 \%$ of ICUs by any physician and in $15 \%$ by ICU staff. The study found that $48 \%$ of physicians who provide overnight staffing had less than 3 months of ICU experience [24].

The direct financial implications of 24/7 in-house coverage can be substantial. Regardless of whether a health system has a salaried or fee-for-service intensivist remuneration system, 24/7 in-house intensivist coverage can be implemented only if significant extra funding is secured to support additional manpower or to compensate for working increased unsociable hours. Additionally, if such coverage is to be implemented universally, this will significantly increase the cost related to ICU admissions, which is already very expensive [22]. Arguably, the system is probably cost-effective by reducing morbidity and mortality, but the evidence of costeffectiveness is lacking at present.

Additionally, in-house $24 / 7$ coverage is perceived to have unfavorable lifestyle implications as it implies working at personally valuable times (evenings, weekends, and nights) [25]. This area has not been studied with the in-house intensivist model, but some inferences can be made from other specialties with shift work. In a survey of 37 pediatric emergency departments, physicians from only eight programs $(22 \%)$ believed they could practice after 50 years of age. Shift work and overnight shifts were given as the most common reason. Stressed physician groups were significantly associated with programs whose attending physicians covered at least $85 \%$ of the night shifts $(P<0.04)$ and reported excessive clinical workload $(P<0.002)$ [26]. Fatigue, exhaustion, and burnout have been attributed to prolonged in-house hours and to night work in residents [22] and intensivists $[27,28]$. Additionally, there are concerns that such a system may make the critical care specialty a less desirable career option, which may further confound the already existing intensivist shortage.

Furthermore, the educational impact of removing the residents and fellows from being the main frontline decision makers is yet to be studied against the value of having immediate supervision around the clock by a qualified physician.

Other manpower alternatives of in-house intensivists, such as hospitalists [29], anesthesiologists [2], and nurse practitioners [22,30], should also be explored. Having an in-house after-hours hospitalist-based system with intensivist off-site back-up was shown to reduce mortality and length of stay compared with a resident-based system in a pediatric ICU [29]. However, hospitalist-based versus intensivist-based systems have not been compared. Additionally, protocolized ICU and tele-ICU have been proposed as alternatives to $24 / 7$ intensivist coverage. In one study, protocolized ICU care driven by intensivist rounding during the day was associated with the same mortality and morbidity as 24-hour in-house intensivist coverage [31]. The application of tele-ICU technology represents an exciting alternative for in-house intensivist coverage [32], with emerging evidence suggesting that the implementation of a tele-ICU program is associated with improved clinical outcomes and hospital financial performance [33].

\section{Conclusions}

Although $24 / 7$ in-house intensivist coverage potentially has several clinical, educational, financial, and occupational implications, our primary focus should be patient outcome. Studies have demonstrated that having an intensivist improves patient outcome [34-37] and that ICU staffing intensity is associated with better patient outcome [38]. In the last two or three decades, the young critical care profession has made several milestones by introducing the intensivist concept and closed-unit model. Having an intensivist has been considered 'the most effective intervention to improve survival of the critically ill that has been devised in the past 30 years [39], and critical care medicine has become a focus of national interest in several countries. Twenty-four/seven inhouse coverage gives all ICU patients the opportunity of being managed by a qualified intensivist at the most critical time of their illness and represents a natural progression of the profession. Therefore, I would recommend 24/7 in-house intensivist coverage for tertiary-care ICUs like the ICU under question. Data are still awaited but, like having an intensivist [40], 24/7 in-house coverage is likely to be cost-effective, especially in large ICUs, and is likely to result in enhanced critical care training. I would also recommend appropriate financial compensation and creative scheduling to reduce the lifestyle burden of 24/7 in-house coverage and to ensure continuity of care [41]. Critical care societies should cam- 
paign for this model as an initiative for patient safety and quality improvement.

\section{Competing interests}

The author declares that he has no competing interests.

\section{References}

1. Arabi Y, Alshimemeri A, Taher S: Weekend and weeknight admissions have the same outcome of weekday admissions to an intensive care unit with onsite intensivist coverage. Crit Care Med 2006, 34:605-611.

2. Luyt CE, Combes A, Aegerter P, Guidet B, Trouillet JL, Gibert C, Chastre J: Mortality among patients admitted to intensive care units during weekday day shifts compared with 'off' hours. Crit Care Med 2007, 35:3-11.

3. Rivers E, Nguyen B, Havstad S, Ressler J, Muzzin A, Knoblich B, Peterson E, Tomlanovich M: Early goal-directed therapy in the treatment of severe sepsis and septic shock. $N$ Engl J Med 2001, 345:1368-1377.

4. Kumar A, Roberts D, Wood KE, Light B, Parrillo JE, Sharma S, Suppes R, Feinstein D, Zanotti S, Taiberg L, Gurka D, Kumar A, Cheang M: Duration of hypotension before initiation of effective antimicrobial therapy is the critical determinant of survival in human septic shock. Crit Care Med 2006, 34:1589-1596.

5. loachimescu OC, loachimescu AG, lannini PB: Severity scoring in community-acquired pneumonia caused by Streptococcus pneumoniae: a 5-year experience. Int J Antimicrob Agents 2004, 24:485-490.

6. Bell CM, Redelmeier DA: Mortality among patients admitted to hospitals on weekends as compared with weekdays. N Engl J Med 2001, 345:663-668.

7. Cram P, Hillis SL, Barnett M, Rosenthal GE: Effects of weekend admission and hospital teaching status on in-hospital mortality. Am J Med 2004, 117:151-157.

8. Uusaro A, Kari A, Ruokonen E: The effects of ICU admission and discharge times on mortality in Finland. Intensive Care Med 2003, 29:2144-2148.

9. Arias Y, Taylor DS, Marcin JP: Association between evening admissions and higher mortality rates in the pediatric intensive care unit. Pediatrics 2004, 113:e530-534.

10. Barnett MJ, Kaboli PJ, Sirio CA, Rosenthal GE: Day of the week of intensive care admission and patient outcomes: a multisite regional evaluation. Med Care 2002, 40:530-539.

11. Ensminger SA, Morales IJ, Peters SG, Keegan MT, Finkielman JD, Lymp JF, Afessa B: The hospital mortality of patients admitted to the ICU on weekends. Chest 2004, 126:1292-1298.

12. Kostis WJ, Demissie K, Marcella SW, Shao YH, Wilson AC Moreyra $A E$ : Weekend versus weekday admission and mortality from myocardial infarction. N Engl J Med 2007, 356:10991109.

13. Higgins TL, McGee WT, Steingrub JS, Rapoport J, Lemeshow S, Teres D: Early indicators of prolonged intensive care unit stay: impact of illness severity, physician staffing, and pre-intensive care unit length of stay. Crit Care Med 2003, 31:45-51.

14. Pollack MM, Cuerdon TT, Patel KM, Ruttimann UE, Getson PR, Levetown M: Impact of quality-of-care factors on pediatric intensive care unit mortality. JAMA 1994, 272:941-946.

15. Jacobs $M$, Hussain $E$, Hall $M$ : Stranger in a strange land: internists in cardiothoracic intensive care. New Horiz 1999, e1-e7.

16. Gajic $O$, Afessa B, Hanson AC, Krpata T, Yilmaz M, Mohamed SF, Rabatin JT, Evenson LK, Aksamit TR, Peters SG, Hubmayr RD, Wylam ME: Effect of 24-hour mandatory versus on-demand critical care specialist presence on quality of care and family and provider satisfaction in the intensive care unit of a teaching hospital. Crit Care Med 2008, 36:36-44.

17. Hixson ED, Davis S, Morris S, Harrison AM: Do weekends or evenings matter in a pediatric intensive care unit? Pediatr Crit Care Med 2005, 6:523-530.

18. Haupt MT, Bekes CE, Brilli RJ, Carl LC, Gray AW, Jastremski MS, Naylor DF, PharmD MR, Md AS, Wedel SK, Md MH; Task Force of the American College of Critical Care Medicine, Society of Critical Care Medicine: Guidelines on critical care services and personnel: recommendations based on a system of categorization of three levels of care. Crit Care Med 2003, 31:26772683.
19. Joint Commission: The ICU and the levels of care. In Improving Care in the ICU. Oak Brook, IL: Joint Commission Resources; 2004:43.

20. Wunsch H, Mapstone J, Brady T, Hanks R, Rowan K: Hospital mortality associated with day and time of admission to intensive care units. Intensive Care Med 2004, 30:895-901.

21. Zimmerman JE, Shortell SM, Knaus WA, Rousseau DM, Wagner DP, Gillies RR, Draper EA, Devers K: Value and cost of teaching hospitals: a prospective, multicenter, inception cohort study. Crit Care Med 1993, 21:1432-1442.

22. Tucci M, Lacroix J: PICU mortality: is it better 9 to $\mathbf{5}$ ? Pediatr Crit Care Med 2005, 6:608-609.

23. Angus DC, Shorr AF, White A, Dremsizov TT, Schmitz RJ, Kelley MA: Critical care delivery in the United States: distribution of services and compliance with Leapfrog recommendations. Crit Care Med 2006, 34:1016-1024.

24. Parshuram CS, Kirpalani H, Mehta S, Granton J, Cook D: Inhouse, overnight physician staffing: a cross-sectional survey of Canadian adult and pediatric intensive care units. Crit Care Med 2006, 34:1674-1678.

25. Zwemer FL Jr., Schneider S: The demands of 24/7 coverage: using faculty perceptions to measure fairness of the schedule. Acad Emerg Med 2004, 11:111-114.

26. Losek JD: Characteristics, workload, and job satisfaction of attending physicians from pediatric emergency medicine fellowship programs. Pediatric Emergency Medicine Collaborative Research Committee. Pediatr Emerg Care 1994, 10: 256-259.

27. Curtis JR, Puntillo K: Is there an epidemic of burnout and posttraumatic stress in critical care clinicians? Am J Respir Crit Care Med 2007, 175:634-636.

28. Embriaco N, Azoulay E, Barrau K, Kentish N, Pochard F, Loundou A, Papazian L: High level of burnout in intensivists: prevalence and associated factors. Am J Respir Crit Care Med 2007, 175: 686-692.

29. Tenner PA, Dibrell H, Taylor RP: Improved survival with hospitalists in a pediatric intensive care unit. Crit Care Med 2003, 31:847-852.

30. Hixson ED, Davis S, Harrison AM: Pediatric intensive care unit mortality: is it better 9 to 5? Pediatr Crit Care Med 2006, 7: 188-189.

31. Handman D, Bank M, Safford M: 24-hour in-house intensivists versus protocolized care in a surgical intensive care unit. Crit Care Med 2004, 32:A30.

32. Leong JR, Sirio CA, Rotondi AJ: elCU program favorably affects clinical and economic outcomes. Crit Care 2005, 9:E22.

33. Rosenfeld BA, Dorman T, Breslow MJ, Pronovost $P$, Jenckes $M$, Zhang $\mathrm{N}$, Anderson $\mathrm{G}$, Rubin $\mathrm{H}$ : Intensive care unit telemedicine: alternate paradigm for providing continuous intensivist care. Crit Care Med 2000, 28:3925-3931.

34. Blunt MC, Burchett KR: Out-of-hours consultant cover and case-mix-adjusted mortality in intensive care. Lancet 2000 356:735-736.

35. Goh AY, Lum LC, Abdel-Latif ME: Impact of $\mathbf{2 4}$ hour critical care physician staffing on case-mix adjusted mortality in paediatric intensive care. Lancet 2001, 357:445-446.

36. Dimick JB, Pronovost PJ, Heitmiller RF, Lipsett PA: Intensive care unit physician staffing is associated with decreased length of stay, hospital cost, and complications after esophageal resection. Crit Care Med 2001, 29:753-758.

37. Pronovost PJ, Jenckes MW, Dorman T, Garrett E, Breslow MJ, Rosenfeld BA, Lipsett PA, Bass E: Organizational characteristics of intensive care units related to outcomes of abdominal aortic surgery. JAMA 1999, 281:1310-1317.

38. Pronovost PJ, Angus DC, Dorman T, Robinson KA, Dremsizov TT, Young TL: Physician staffing patterns and clinical outcomes in critically ill patients: a systematic review. JAMA 2002, 288: 2151-2162.

39. Durbin CG Jr.: Team model: advocating for the optimal method of care delivery in the intensive care unit. Crit Care Med 2006, 34:S12-17.

40. Pronovost PJ, Needham DM, Waters H, Birkmeyer CM, Calinawan $\mathrm{JR}$, Birkmeyer JD, Dorman T: Intensive care unit physician staffing: financial modeling of the Leapfrog standard. Crit Care Med 2004, 32:1247-1253.

41. Redelmeier DA, Bell CM: Weekend worriers. $N$ Engl J Med 2007, 356:1164-1165. 